\title{
A Platform of Risk Analysis and Early-warning for Urban Road Waterlogging Problem Based on IOI Technology
}

\author{
Fanlei Zeng ${ }^{1,2,3}$, Jianwei Liu ${ }^{4}$, Chongfu Huang ${ }^{1,2,3^{*}}$ \\ ${ }^{1}$ Key Laboratory of Environmental Change and Natural Disaster, Ministry of Education of China, Beijing \\ Normal University, Beijing 100875, China \\ ${ }^{2}$ State Key Laboratory of Earth Surface Processes and Resource Ecology (Beijing Normal University), Beijing \\ 100875, China \\ ${ }^{3}$ Academy of Disaster Reduction and Emergency Management, Ministry of Civil Affairs \& Ministry of Education, the \\ Peoples' Republic of China, Beijing 100875, China \\ ${ }^{4}$ Handan Meteorological Bureau,Handan 056000,China
}

\section{一种基于智联网技术的城市暴雨内港风险分析与预警 平台}

\author{
曾凡雷 ${ }^{1,2,3}$, 刘建伟 ${ }^{4}$, 黄崇福 ${ }^{1,2,3^{*}}$ \\ ${ }^{1}$ 北京师范大学环境演变与自然灾害教育部重点实验室, 北京 100875 , 中国 \\ ${ }^{2}$ 地表过程与资源生态国家重点实验室 (北京师范大学), 北京 100875, 中国 \\ ${ }^{3}$ 民政部教育部减灾与应急管理研究院, 北京 100875 , 中国 \\ ${ }^{4}$ 䢶郸市气象局, 期郸 056000 , 中国
}

\begin{abstract}
Urban waterlogging, which impact traffic and residents directly, is influenced both by meteorological and urban drainage capacity. Urban waterlogging problem is also a complex issue involving meteorological department, the municipal sector, the transport sector and the general public, which may be solved helpful through the integration of information and intelligence. This paper established an urban storm waterlogging risk analysis and early warning platform participated by multiple industry groups with the IOI technology. By monitoring and updating basic data by relevant personnel, we can achieve dynamic assessment of risk. The former platform can assist municipal, transportation and other management department make emergency preparedness in advance, and can help people arrange travel routes in
\end{abstract}

*通讯作者：黄崇福 hchongfu@bnu.edu.cn advance, which helps to avoid dangerous sections, so as to better serve the people's life and production.

Keywords: internet of intelligences; storm waterlogging; risk analysis; early warning platform

\section{摘要}

城市内涝, 直接影响道路交通和居民生活。发生与否, 不仅受气象要素的影响, 更与城市排水能力密切相 关。城市内涝问题, 是一个涉及气象部门、市政部门、 交通部门以及社会公众等多个群体的复杂问题。整合 信息和集成智慧, 有助于解决这一问题。本文利用智 联网技术, 建立了一套可由多个行业群体共同参与的 城市暴雨内涝风险分析与预警平台。通过作为智能体 的相关人员对基础数据的监测与更新, 可以实现对风 险的动态评估。城市暴雨内涝的预警平台可以辅助市 政、交通等管理部门提前做好应急准备, 同时可以帮 助市民提前安排出行路线, 避开危险路段等, 从而较 好的服务于人们的生产生活。 
Risk Analysis and Crisis Response in Big Data Era (RAC-16)

关键词：智联网, 暴雨内涝, 风险分析, 预警平台

\section{1. 引言}

城市内港是目前我国城市化发展进程中的突出问题 ${ }^{[1]}$ ，建设部对全国 350 个城市的一项调查报告显示， 2008-2010 年间, 全国 60\%以上城市发生过城市内涝, 遭受内涝灾害超过 3 次以上的城市有 137 个, 其中 57 个城市的最大积水时间超过 12 小时 ${ }^{[2]} ; 2012$ 年 7.21 北京特大暴雨导致市区内积水点 114 处 ${ }^{[3]}$ 。

城市内涝的发生是由于长时间的降雨或强降雨 超出了城市的排水能力, 从而引发了城市内产生积水 灾害的现象。它不仅对交通、地下管线等城市生命线 系统造成直接的破坏，同时会对城市经济、居民生活 等造成较大影响。对城市内涝灾害的研究是一个涉及 气象部门、市政部门、交通部门以及社会公众等多个 行业群体的复杂问题。在气象灾害防御工作中, 我国 气象部门确立了 “政府主导、部门联动、社会参与” 的工作机制 ${ }^{[4,5]}$, 从制度上确立了 “整合信息、集成 智慧” 的问题解决思路。

智联网技术是一种基于互联网, 通过整合大多数 人的智慧实现智力提升的网络模型, 其目的是为了帮 助人们解决生活中遇到的各种问题 ${ }^{[6]}$ 。自 2011 年提 出以来, 经过近几年的发展 ${ }^{[7-12]}$, 已经有多个应用型 智联网平台问世 ${ }^{[13-18]}$ 。本文利用智联网技术, 建立了 一套可以由气象部门、市政部门、交通部门以及社会 公众等多个行业群体共同参与的城市暴雨内涝风险 分析与预警平台。在移动互联网时代, 可以随时连接 网络并提供信息的人, 就是一个智能的传感器, 通过 智能体对基础数据的监测与更新, 可以实现对风险的 动态评估。本文最后以期部市为例建立了城市暴雨内 涝风险分析与预警的应用平台。

\section{2. 智联网简介}

2011 年黄崇福首次提出智联网的概念 ${ }^{[6]}$, 并给出了智 联网的完整定义:

设 $\mathrm{A}$ 是一个智能体集合, $\mathrm{N}$ 是 $\mathrm{A}$ 使用的一个网络, $M$ 是处理 $A$ 所提供信息的模型, 则三元体 (A, N , M ) 称为一个智联网, 如图 1 所示。

$\mathrm{Q}=\mathrm{R}(\mathrm{A}, \mathrm{N}, \mathrm{M})$

根据智联网的定义, 一个完整的智联网是由网络 $\mathrm{N}$ 、智能体集合 $\mathrm{A}$ 以及信息处理模型 $\mathrm{M}$ 三部分构成的 有机整体, 每个部分都是一系列的集合, 例如, $N$ 表 示由网络硬件、软件等示由网络硬件、软件等构成的 可以互联的网络; $A$ 表示具有一定智力水平的各种实

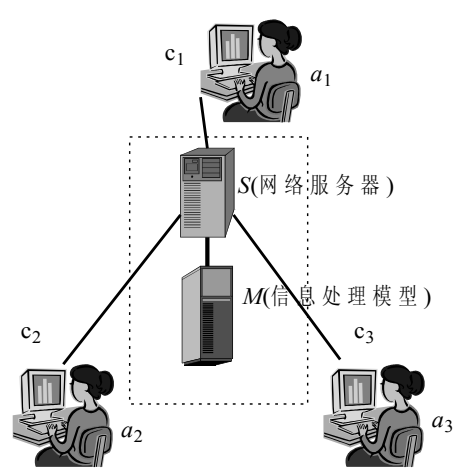

图 1. 最简单的智联网

体, 是智联网中智力的基础, 而人作为具有高等智力 的生物, 是最典型的智能体。而 $M$ 代表智联网的各种 信息处理模型, 针对不同的信息形式, 需要不同的模 型来处理, 它是提升智联网智力水平的关键环节 ${ }^{[11]}$ 。

从而, 智联网的主要功能可描述为: 大量的智能 体通过网络平台贡献个体经验与智慧, 借助于信息处 理模型完成经验融合并实现智力提升, 并用于帮助人 们解决生活中遇到的各种问题。

\section{3. 智联网的服务流程}

智联网通过联合大多数人的智慧实现智力提升, 从而 帮助人们解决生活中的各种问题, 其基本流程包括: 问题发布, 问题推送, 信息收集、模型处理以及经验 分享等五个步骤，具体步骤可描述以下:

(1) 问题发布: 即用户根据自身需求, 在智联 网平台完成问题定制, 并在智联网平台发布。

(2) 问题推送: 智联网根据问题的类型特征, 将问题推送给特定的智能体用户。

（3）经验反馈: 智能体在接收到系统推送的问 题后, 根据自身经历、经验或知识储备等, 向系统平 台反馈相应的经验知识, 经验数据将被保存为数据 库、文件、其他等格式。当大量的智能体参与反馈经 验时, 则智联网平台就实现了对问题的信息收集。

（4）模型处理: 接收到智能体反馈到的经验信 息后, 智联网提供不同的信息处理模型, 实现排除恶 意虚假信息、整合有效信息等操作, 最终得出综合的 经验信息。

(5) 经验分享: 根据步骤 (4) 中得到的综合经 验信息，反馈给客户，用于解答相应的问题。 


\section{4. 智联网技术在城市暴雨内涝问题中的应用}

城市暴雨内涝灾害不仅与气象因子有关, 而且与城 市排水能力有关; 它发生后会直接影响道路交通,

同时会干扰居民的日常生活。因此，城市暴雨内涝 问题是涉及到气象、市政、交通以及公众等多个行 业群体的复杂问题。在智联网中将相关的群体作为 不同专业的智能体，共同参与分析城市内涝风险问 题。

\section{1. 智能体的构成}

笔者将城市暴雨内涝智联网中的智能体分为以下四 类:

（1）气象部门专业智能体: 指气象专业人员。 他们利用专业知识提供气象要素相关数据, 如降雨 强度的预报数据等。

（2）市政排水部门专业智能体：指市政排水部 门专业人员。他们掌握城市内的排水管网的分布以 及排水能力等, 可以提供城市内的易积水路段, 以 及每个路段的排水能力等信息, 尤其在暴雨发生时, 市政排水公司往往会派专门人员观察易涝点的情 况，第一时间掌握每个路段的积水信息。

（3）交通部门专业智能体：指交通部门专业人 员。当城市内涝引发的道路积水达到一定的深度, 他们会执行交通管制, 从而避免城市内涝严重路段 可能造成的安全隐患。

（4）社会公众智能体: 指一般社会人员。他们 的数量较多, 常常在监测社会潜在危险源方面发挥 重要作用, 将会为智联网提供丰富的数据资源。

\section{2. 主要模型}

根据城市暴雨内涝风险问题的实际情况，建立了以 下两类分析模型:

（1）降雨强度-积水深度关系模型（Rain-Depth Model）：该模型是一种根据降雨强度数据估计道路 积水深度的预测模型, 目前类似的预测模型较多, 如（a）通过对城市地形、街道、排水管网、河道等 要素的建模, 模拟不同强度降雨可能产生的积水深 度的仿真模型; （b）通过对易积水路段的积水深度 以及降雨强度历史数据的分析, 建立二者模糊对应 关系的经验预测模型等。

需要说明的是, 任何一种预测模型在建模过程 中都很难完全考虑实际发生过程中的各种情况, 因 此, 根据降雨强度的积水预测结果仅能够作为参考, 在实际应用中, 智能体实时提供的积水数据, 通常 比模型预测的数据更为准确。
（2）积水深度-影响对象风险情景模型: 指不同 积水深度对不同影响对象的风险情景影响关系, 主 要的影响对象包括 “附近建筑”、“过往车辆”、 “过往行人” 等。如积水达到 20 厘米步行困难, 超 过 25 厘米将采取交通管制, 超过 30 厘米自行车和 小汽车行进困难等。其中, 附近建筑主要考虑积水 深度与建筑的台阶高度的关系, 影响后果以建筑进 水程度为标准, 过往车辆以车辆排气筒高度为评价 指标, 分析不同积水深度对不同车型的影响, 过往 行人主要考虑积水深度、路坑、排水井、裸露电线 等危险源, 分析不同积水深度对行人察觉危险源能 力的影响。

\section{3. 平台架构}

平台结构共分为四层, 分别为用户层、表示层、模 型层以及数据层, 如图 2 所示。

用户层主要是指由气象部门、市政排水部门、 交通部门以及社会公众等不同行业的智能体构成的 结构层, 在城市内涝智联网中, 用户层既是基础数 据的提供者, 也是风险预警信息的接收者。

表示层是与用户层直接交互的结构层, 主要是 智能体反馈信息的人机交互界面以及预警信息的展 示界面等。

模型层主要是智联网中根据实际问题提供的各 种模型, 如用于城市内涝风险分析的易涝点 “雨强水深关系模型”、“水深-承受体影响关系模型” 等。 数据层中以数据库的方式保存了各种基础数 据, 如智能体数据库、降雨历史记录数据库、道路 积水事件数据库、次生危险源数据库等。

\section{4. 主要功能}

（1）基础数据收集功能: 主要包括易涝点积水 事件收集、潜在危险源信息收集, 道路积水后主要 受影响社区或商店信息收集以及降雨强度信息的收 集等。其中, 降雨强度信息由气象部门专业人员提 供, 可以文件方式直接读取, 其他数据由市政排水 人员、社会公众等以网页交互的方式提供, 如图 3 所示。

（2）信息整合功能: 基于不同的信息处理模型, 对多个通过有效性评估的经验信息进行整合, 得出 较为完整以及合理的综合信息, 如图 4 展示了 3 个 智能体反馈的积水事件经验信息经过模型处理后的 综合积水事件经验信息。 
Risk Analysis and Crisis Response in Big Data Era (RAC-16)

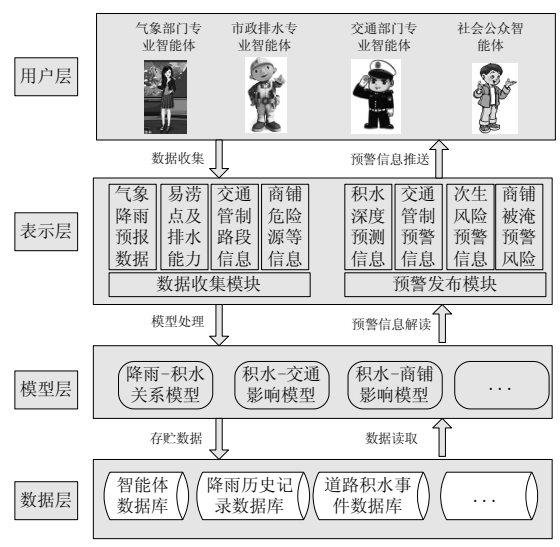

图 2. 城市暴雨内涝智联网平台架构

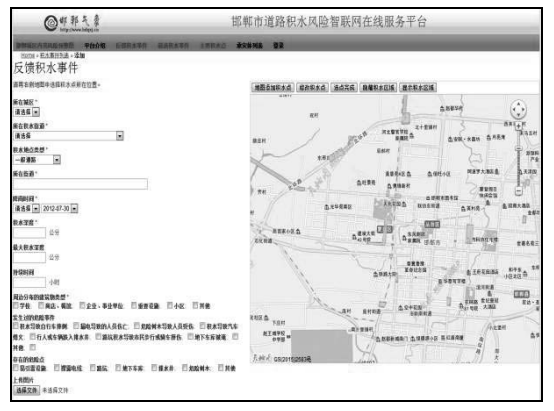

图 3. 社会公众智能体反馈积水事件信息界面

（3）预警信息的发布功能: 采用了在线地图的 展示方式, 以地理位置以及不同颜色为标记, 展示 可能出现积水的路段的预警信息, 包括易涝点积水 深度预警（图 5)、积水路段附近的潜在受影响对象 预警（图 6) 等。

\section{5. 结论和讨论}

本文研究了利用智联网技术研究城市内涝风险分析 与预警的方法。提出一种由气象部门、市政排

水部门、交警部门以及社会公众等共同参与的 城市内涝风险研究方法, 借助于智联网平台, 不同 行业的智能体可以发挥各自的专业特长, 从而很好 的为城市内涝风险研究服务。借助于在线地图的方 式, 可以更加直观的展示与地理位置有关的道路积 水风险信息。该预警平台已经投入试用，效果良好。

该平台通过智能体参与, 可以随时搜集来自于 互联网的信息, 具有快速更新数据, 动态跟踪风险

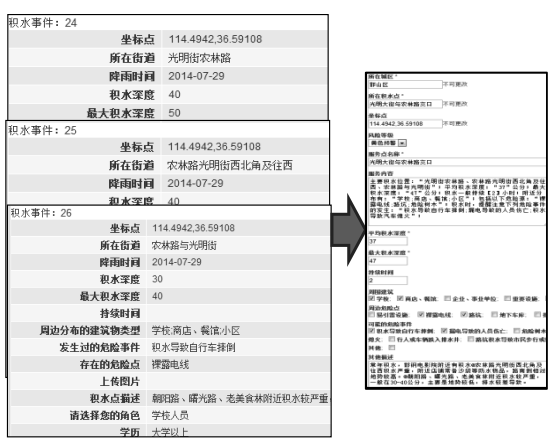

图 4. 零散经验信息的综合

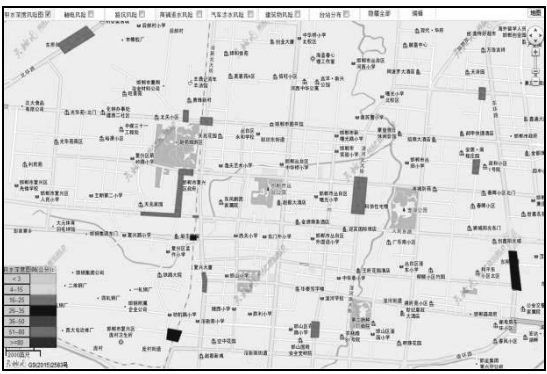

图 5. 邯単市易涝路段积水深度预警图

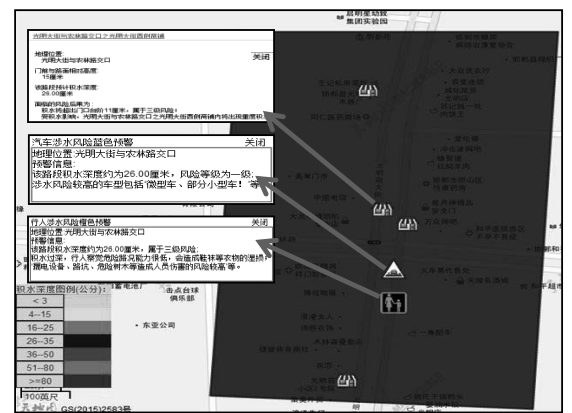

图 6. 邯郸市某易涝点的道路积水风险预警图

的特点。根据积水预报模型提前发布的预警信息, 可以帮助市政、交警等部门提前做好城市内涝发生 的准备, 帮助市民提前规划好出行路线。通过智能 体现场监测反馈的道路积水信息, 可以较快的更新 到在线地图中, 进一步为市政、交警等管理部门提 供决策支持, 帮助市民随时调整出行路线等。由于 篇幅有限, 本文仅就智联网的核心功能做了简要介 
绍, 智联网的其他关键技术如经验信息的表达方法、 智能体的激励机制、恶意虚假信息的甄别策略等并 未提及, 但是也尤为重要, 相关内容的探讨可参考 文献 ${ }^{[10][11]}$ 。

\section{Acknowledgements}

This paper was supported by National Natural Science Foundation of China (No. 2012CB955402), the State Key Development Program for Basic Research of China (973 project) (No. 2012CB955402), project from Department of Science in Hebei (No. 16275410D), and project from Handan Meteorological Bureau (No. 14hd10).

\section{致谢}

本研究得到了国家自然科学基金项目 (41471424)、国家重大科学研究计划(2012CB955402)、 河北省科技厅项目（16275410D）以及邯郸市气象局 项目（14hd10）的支持。

\section{参考文献}

[1] 鲁航线等.城市防洪、排涝及排水三种设计标准的 关系初探. 城市道桥与防洪, 2007, (11) : 64-66

[2] 凤凰网, 中国为何治不好城市内涝. http://news.ifeng.com/opinion/special/neilao/

[3] 中国天气网, 天气灾害大事记. http://www.weather.com.cn/zt/kpzt/696656.shtml

[4] 中国气象局,机制推动: 政府主导部门联动社会参 与.

http://www.cma.gov.cn/2011xzt/2012zhuant/20120 710/2012071006/201207/t20120713_178392.html

[5] 华兴夏,苏向荣. 论公众参与公共气象服务的重要 意义．阅江学刊, 2013, 6: 60-66.

[6] C.F. Huang, Internet of Intelligences in Risk Analysis for Online Services, Journal of Risk Analysis and Crisis Response, 1(2): 110-117, 2011

[7] C.F. Huang. A paradigm to construct Internet of intelligences for risk assessment. Emerging Economies, Risk and Development, and Intelligent Technology, Eds. C.F. Huang, A. Lyhyaoui, G.F. Zhai and N. Benhayoun . Boca Raton, USA: CRC Press, pp. 1-6, 2015.

[8] 黄崇福. 风险分析的困惑和智联网的使命. 风险分 析和危机反应的创新理论和方法. 黄崇福, 翟国 方编. 巴黎:Atlantis 出版社, pp.1-9, 2012.

[9] C.F. Huang, The measurement of effective knowledge in multiple Internet of intelligences and application in risk assessment. Intelligent Systems and Decision Making for Risk Analysis and Crisis Response, Eds. C.F. Huang, C. Kahraman. Boca Raton, USA: CRC Press, pp. 1-8, 2013

[10]F.L. Zeng, C.H. Huang, F.L. Ai. Operation mechanism and interfered information's screening strategy of internet of intelligence, Intelligent Systems and Decision Making for Risk Analysis and Crisis Response, Eds. C.F. Huang, C. Kahraman. Boca Raton, USA: CRC Press, pp. 313-318, 2013

[11]C.F. Huang, Multiple Internet of intelligences for risk analysis, Journal of Risk Analysis and Crisis Response, 2014, 4(2): 61-71.

[12]艾福利. 自然灾害风险分析智联网服务平台构建 与应用研究.北京师范大学博士学位论文, 2013

[13] 艾福利, 黄崇福, 王蔚丹. 高考志愿填报风险评估 智联网服务平台. 风险分析和危机反应的创新理 论和方法. 黄崇福, 翟国方编. 巴黎: Atlantis 出 版社, pp. 44-49, 2012.

[14]王蔚丹,黄崇福,艾福利.基于智联网的地震预报策 略初探. 风险分析和危机反应的创新理论和方法. 黄崇福, 翟国方编. 巴黎: Atlantis 出版社, pp. $50-55,2012$.

[15] T. Wu, C.F. Huang, F.L. Ai. A discussion on using internet of intelligences to improve risk radar. Intelligent Systems and Decision Making for Risk Analysis and Crisis Response, Eds. C.F. Huang, C. Kahraman. Boca Raton, USA: CRC Press, pp. 831-837, 2013

[16] 北京崇安智联科技服务有限责任公司. 高考志愿 填报智联网服务平台一联接智慧, 网获知识. http://www.cazl.cn/ioiserver/index.php?r=site/main

[17] 北京崇安智联科技服务有限责任公司. 温州台风 灾害风险分析智联网. http://www.cazl.cn/ioi4ndra

[18] W.D. Wang, S. Qiao, F.L. Zeng, J. Guo. An approach to ranking integrated models for risk assessment by using the internet of intelligences. Journal of Risk Analysis and Crisis Response, 2016, 6(1): 2-9. 